This item was submitted to Loughborough's Research Repository by the author.

Items in Figshare are protected by copyright, with all rights reserved, unless otherwise indicated.

\title{
Low frequency induction heating for the sealing of plastic microfluidic
} systems

\section{PLEASE CITE THE PUBLISHED VERSION}

http://dx.doi.org/10.1007/s10404-009-0539-x

PUBLISHER

(C) Springer-Verlag

VERSION

AM (Accepted Manuscript)

LICENCE

CC BY-NC-ND 4.0

\section{REPOSITORY RECORD}

Knauf, Benedikt J., D. Patrick Webb, Changqing Liu, and Paul P. Conway. 2019. "Low Frequency Induction Heating for the Sealing of Plastic Microfluidic Systems". figshare. https://hdl.handle.net/2134/7705. 
This item was submitted to Loughborough's Institutional Repository (https://dspace.lboro.ac.uk/) by the author and is made available under the following Creative Commons Licence conditions.

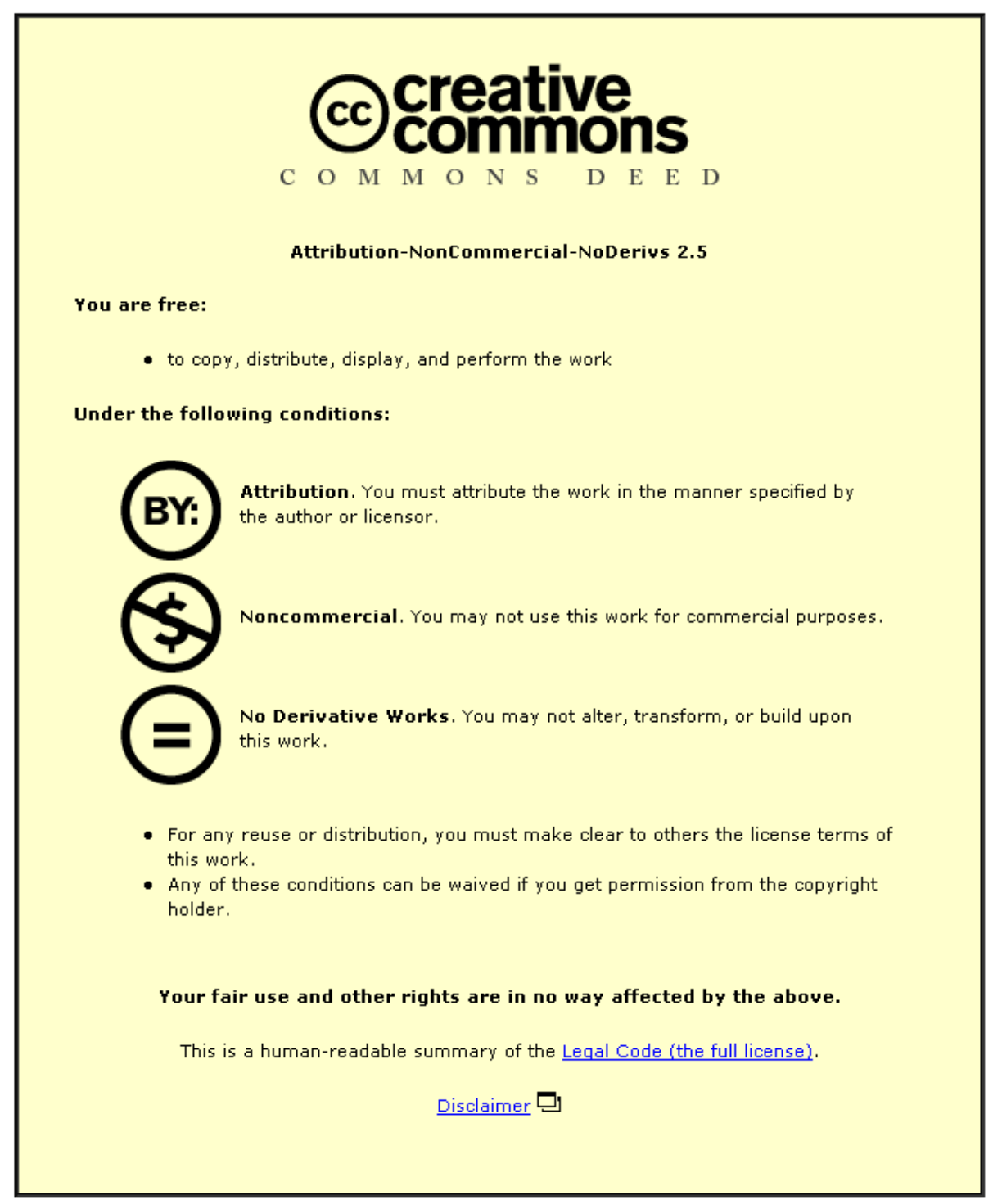

For the full text of this licence, please go to: http://creativecommons.org/licenses/by-nc-nd/2.5/ 


\title{
Low Frequency Induction Heating (LFIH) for the Sealing of Plastic Microfluidic Systems
}

\author{
Wolfson School of Mechanical and Manufacturing Engineering, Loughborough University, \\ Loughborough, LE11 3TU, UK \\ b.j.knauf@1boro.ac.uk,
}

Phone: +44 (0) 1509227677

Fax: $+44(0) 1509227648$

\begin{abstract}
Microfluidic systems are being used in many applications and the demand for such systems has been phenomenal in past decades. To meet such high volume market needs, a cheap and rapid method for sealing these microfluidic platforms which is viable for mass manufacture is highly desirable. Low frequency induction heating has been introduced as potential basis of a cost-effective, rapid production method for polymer microfluidic device sealing in preceding publications. Through this technique excellent bond strength was achieved, withstanding an air-pressure of up to $590 \mathrm{kPa}$. However, it has been found that during the bonding process it is important to effectively manage the heat dissipation to prevent distortion of the microfluidic platform. The heat affected zone, and the localised melted area, must be controlled to avoid blockage of the microfluidic channels or altering the channels' wall characteristics. This work presents an analytical approach to address the issues and provide a basis for process optimisation and design rules.
\end{abstract}

Keywords Microfluidic Systems, Induction heating, Plastic bonding

\section{Introduction}

As micro systems are getting smaller and smaller while demand for their use in harsh environments is growing, the requirements on the packaging are becoming ever more stringent. The packaging must protect the system against dirt, humidity, stresses, etc and, depending on the application, it has to embrace electrical, fluidic or optical interconnection and thermal management.

A specific task is the packaging of microfluidic systems. Microfluidic systems are networks of channels with width and depth in the micron scale, designed to do continuous flow chemistry with small volumes of fluid. Microfluidic devices are also referred to as lab-on-a-chip (LOC) or, if they are more complex, micro total analysis systems ( $\mu \mathrm{TAS}$ ). In this application packaging must not only withstand external influences, but also internal pressures. The world-to-chip interface, the interconnection for scaling the fluid delivery network from macro down to micro dimensions and coupling them into the microfluidic system, are also highly complex compared to those of "normal" MEMS. These interfaces have to be strong and flexible, must provide good sealing, and must connect reservoirs of millilitre or litre volumes to systems with a capacity of micro or even nanolitres.

Most of the existing sealing and interconnecting techniques are cost-intensive and slow compared to the other manufacturing steps of the microfluidic system, so that they pose a bottleneck in mass production. Hence, rapid and cheap techniques to seal and interconnect microfluidic chips are highly desirable. A single technique being able to do both sealing and interconnecting, either sequentially or even simultaneously, would be the optimum solution.

In principle, microfluidic systems are platforms containing microfluidic channel networks with a lid sealing those channels. Lid and platform have a thickness of a few $\mathrm{mm}$ while the channels in the platform can have a width and depth smaller than $100 \mu \mathrm{m}$. To be able to access the microfluidic channels holes with a diameter of about $500 \mu \mathrm{m}$ are drilled into the lid working as ports for micro tubing.

The materials being used for microfluidic devices depend on the applications. Glass is chemically inert but it is expensive and hard to process. Silicon can be used to make active parts like micro valves or pumps but it is also expensive. Polymers are capable only of passive channel networks, but due to being cheap in acquisition and easy to process, they are suited to mass manufacture.

Low frequency induction heating (LFIH) has been identified as a technique for the sealing and packaging of polymer microfluidic systems in preceding publications (Knauf et al. 2008). Induction heating is well established in the steel industry for hardening, melting, soldering, welding, and annealing (Anonymous 1993), and increasingly is finding application in other areas like heating fillings in dental medicine, and bottle cap sealing (Cheltenham Induction Heating Ltd 2006). Induction 
heating is used for rapid temperature variations during micro injection moulding to achieve structures with high aspect ratio (Chen et al. 2006) and for solder bonding for MEMS packaging (Yang et al. 2003). Induction welding has already been used for joining of thermoplastics but rather in macro scale (Stokes et al. 2003). The performance of susceptor materials in magnetic fields has been studied before (Yang et al. 2006) and general dependency between change of temperature and heating parameters was identified (Nichols et al. 2006) but the derivation of an applicable analytical model was missing.

The advantages of this technique are manifold. It is a very rapid heating process with a small scaling loss, the start-up is very fast, it is energy efficient, and the technique is capable of high production rates. LFIH bonding is able to achieve good bond strength while avoiding distortion in the microfluidic platform. However, in the joining process it is inevitable that the heat is generated in the susceptor layer which is then dissipated in the surrounding polymer. Therefore it becomes critical to manage the heat dissipation. In this work an analytical approach to controlling the heat dissipation is presented to provide a basis for process optimisation and design rules.

\subsection{Induction Heating}

The discovery of electromagnetic induction by Michael Faraday in 1831 led to the development of electric motors, generators, transformers and wireless communications devices. All the time heat loss was a major factor reducing the efficiency of these systems and researchers sought to minimize it. In the early $20^{\text {th }}$ century the heat loss was utilized for the first time. This utilization was referred to as induction heating and nowadays it is used for many different applications.

The main benefits over other heating methods are the selectivity of the heated area, the fast response time, and a good efficiency.

An induction heating unit consists of a power generator, a tank circuit with coil and a water cooling system. The tank circuit, which is connected to the power generator and the cooling system, provides power and cooling connections for the coil and is equipped with four or more capacitors. These capacitors are connected to the coil to create a resonant circuit. During calibration the power generator checks the resonance frequency of this circuit and generates an alternating current of the same frequency. Then the work piece is put into the magnetic field of the coil to be heated.

\subsubsection{Physical Principles}

The fundamental theory of induction heating is similar to that for transformers. The work coil used in induction heating is equivalent to the first coil in a transformer, while the load functions as a shortwired second coil.

The premise of induction is that a change in magnetic flux induces a current in a circuit or conductor. The change in magnetic flux can be achieved by either altering the magnetic field or moving the conductor in the magnetic field. The principle is expressed by Faraday's law:

$$
E=-N \frac{d \varphi}{d t}
$$

Where

$E$ : Induced voltage

$N$ : Number of windings of the coil

$\Phi$ : Magnetic flux through a single winding

T: Time

The minus sign means that the induced voltage $E$ will cause a current to flow that generates a magnetic field counteracting the change in the inducing field (Lenz's Law).

Every conductor offers resistance to a flow of a current which causes loss of power. The loss of power is converted to heat energy and is described in Joule's law:

$P=R \cdot I^{2}$

Where

$P$ : $\quad$ Power dissipated in the conductor

$R: \quad$ Resistance of the conductor

I: $\quad$ Current induced in the conductor

This effect is also referred to as the Joule effect. In most induction heating applications there is a nonuniform distribution of current induced in the conductor. Equation 2, however, gives us an idea of which parameters affect the heating rate.

For ferromagnetic materials in an alternating magnetic field a second heating effect occurs. The magnetic orientation of the domains of the susceptor (a metal component that absorbs energy from the induction field) aligns with and attempts to follow the rapidly varying field. The friction of this movement in the crystal plane heats up the metal and is referred to as hysteresis heating. If a ferromagnetic material is heated to its Curie temperature it becomes paramagnetic and hysteresis heating ceases.

Alternating currents tend to flow preferentially on the outside of a conductor. This "skin-effect" is characterized by its penetration depth $\delta$.

The penetration depth is defined as the thickness of the layer, measured from the outside surface, in which $87 \%$ of the power is developed (Callebaut 2007).

For an alternating current of frequency $f$ the penetration depth is given by 
$\delta=\sqrt{\frac{2 \cdot \rho}{\mu \cdot \omega}}$

(Davis and Simpson 1979)

$\Rightarrow \delta=\sqrt{\frac{\rho}{\pi \cdot \mu \cdot f}}$

$$
\begin{array}{ll}
\text { Where } \\
\delta: & \text { Penetration depth } \\
\rho: & \text { Resistivity } \\
\mu: & \text { Permeability } \\
f: & \text { Frequency }
\end{array}
$$

Permeability $\mu$ is the product of the magnetic field constant $\mu_{0}$ and the relative permeability $\mu_{r}$ of the conductor

$$
\begin{aligned}
& \mu=\mu_{0} \cdot \mu_{r} \\
& \mu_{r}=\frac{B}{B_{0}}
\end{aligned}
$$

\section{Where}

$B$ : Magnetic flux density in the conductor $\left[\mathrm{Vs} / \mathrm{m}^{2}\right]$ $B_{0}$ : Magnetic flux density in vacuum $\quad\left[\mathrm{Vs} / \mathrm{m}^{2}\right]$

$\mu_{r}$ is less than 1 for diamagnetic materials, and slightly greater than 1 and many times greater than 1 for paramagnetic and ferromagnetic materials, respectively.

Efficiency of the heating process drops when the workpiece is too thin but in order to heat the susceptor homogeneously its thickness should be smaller than the penetration depth $\delta$. At a frequency of $220 \mathrm{kHz}$, which was used during this work, the penetration depth at room temperature is $12 \mu \mathrm{m}$ for nickel, $14 \mu \mathrm{m}$ for steel, $143 \mu \mathrm{m}$ for copper, and 552 $\mu \mathrm{m}$ for aluminium.

\subsubsection{Coil Design}

The design of the coil is one of the most important aspects of induction heating. It defines how the magnetic flux is coupled into the work piece, where the hotspots are (if any), which areas are affected by induction heating, etc. Every design has a different inductivity. The coil and capacitors in the tank circuit form a resonant circuit in which an alternating current is driven at the resonant frequency by the power supply. The voltage output from the power supply is varied to attain a desired output power with a given coil / capacitors combination. According to S. Zinn and S.L. Semiatin coil design is generally based on experience and empirical data rather than simulations (Zinn and Semiatin 1988). This statement was confirmed to the authors by a source working for an induction heating equipment manufacturer (Hüttinger Elektronik).
The coils can be designed with single or multiple turns. In addition to these "standard" coils there are endless variations designed for special purposes. Zinn and Semiatin addressed some basic design considerations to improve efficiency. The distance between the windings should be kept as small as possible and the work piece should be as close as feasible to the coil to assure maximum energy transfer. As the magnetic centre of the inductor is not necessarily its geometric centre the work piece should be rotated to gain more homogeneous heat distribution. The coil also must be designed to prevent cancellation of the magnetic field. If two windings running in opposite directions are too close to each other each associated magnetic field will be cancelled by the other.

For this work a flat helix coil was used. This kind of coil showed a very good heating rate for thin susceptor layers during preliminary experiments (Knauf et al. 2008) and has the additional benefit of being easy to access.

\section{Initial Experimental Trials}

To join plastics with LFIH a susceptor placed at the joining interface can be used. Hence heat is delivered directly to the joining interface, reducing the potential for heat distortion in the part.

In initial feasibility trials (Knauf et al. 2008) PMMA plates $2 \mathrm{~mm}$ thick and $25 \mathrm{~mm} \times 25 \mathrm{~mm}$ in area were joined by locating a thin film susceptor between the plates, clamping and heating in an induction field. Two methods were used of placing a susceptor. Firstly a $5 \mu \mathrm{m} \mathrm{Ni}$ film was plated on evaporated seed layer, covering the whole of the surface on one plate. Secondly a $7.5 \mu \mathrm{m}$ thick Ni foil, $15 \mathrm{~mm} \times 15 \mathrm{~mm}$ in area was clamped between the plates.

Visible melting of the polymer and strong plastic to plastic joints were formed in seconds with use of the $7.5 \mu \mathrm{m}$ nickel foil as susceptor. The joints were pressure tested with air to 5.9 bar without failing. The $5 \mu \mathrm{m}$ plated nickel coating was also found to heat sufficiently to melt the PMMA. However, because the $\mathrm{Ni}$ layer covered the whole of the surface of one of the plates no plastic-plastic bond was formed, and the plastic-metal bond was found to be weak. Thus in order to create bonds between the two plastic substrates directly the optimum susceptor design is a thin metal track following the microfluidic channels on both sides, as shown in Fig. 1, allowing plastic - plastic bonds to be formed. 


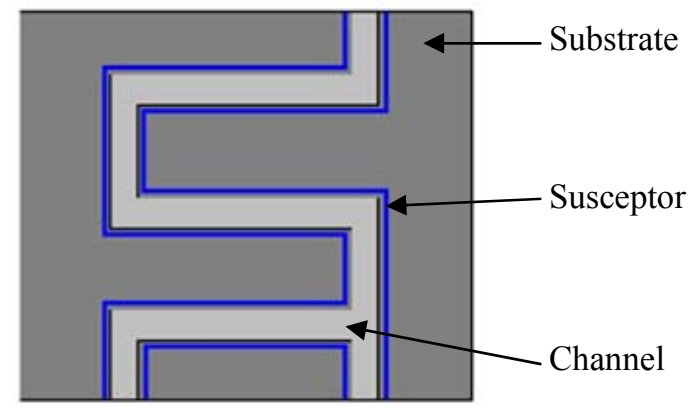

Fig. 1 Metal tracks following microfluidic channels

The design of the metal tracks still has to be optimised. For the design shown in Fig. 1 there is no bond on the substrate's borders. To increase the chip's stability the tracks should follow the substrate's corners as well. This would also increase efficiency of the heating process. Although it is possible to heat open tracks a realistic design would be a series of closed loops to increase coupling strength.

Because it is desirable that the susceptor be as thin as possible, a 50-100 nm evaporated nickel coating was also tested and was found to heat but not sufficiently to melt the PMMA. While ferromagnetic materials such as nickel have the strongest coupling to the induction field, a $5 \mu \mathrm{m}$ thick coating of sputtered aluminium which is paramagnetic was also found to heat rapidly.

$7.5 \mu \mathrm{m}$ thick nickel rings with an inner diameter of $6 \mathrm{~mm}$ and outer diameter of $9 \mathrm{~mm}$ were used for later experiments. These experiments were designed for characterising the process in terms of bond strength, heat affected zone and melted area. Fig. 2 shows a sample for pressurising tests:

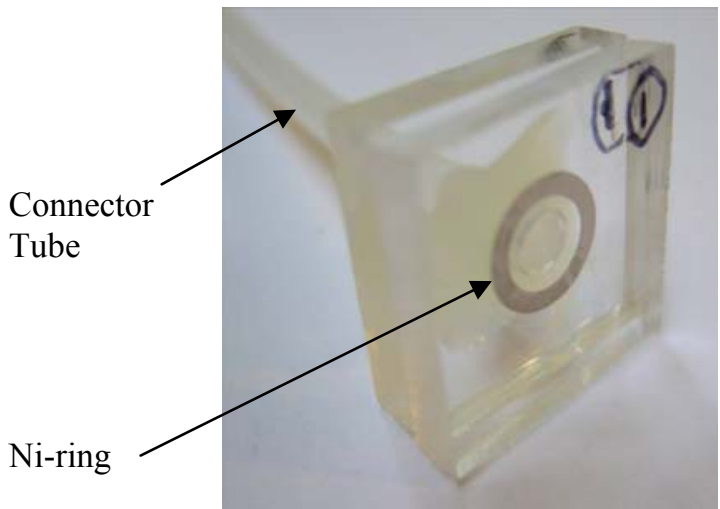

Fig. 2 Ni-ring used to bond PMMA substrates

For some test samples the melt area around the ring was observed to be up to $1 \mathrm{~mm}$ in width. In order to optimize the bonding process for microfluidic applications this melt area has to be reduced as much as possible while still offering good bond strength. Hence a greater understanding of the induction bonding process and the heat dissipation is needed. The work presented in this paper concentrates on an analytical approach to enable the prediction of the amount of heat delivered to the work piece during bonding processes.

\section{Analytical and Experimental Approaches}

\subsection{Arrangement Modelled}

For analytical modelling of the induction heating process certain design parameters had to be assumed. The workpiece was chosen to be a ring placed in the magnetic field of a single loop inductor, coaxial with the inductor (see Fig. 3).

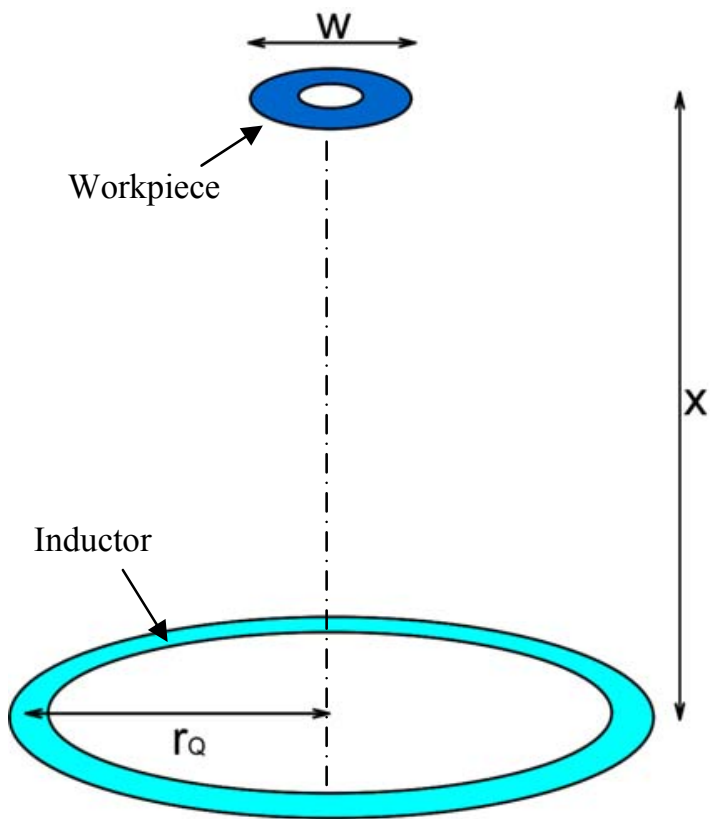

Fig. 3 Assumptions for analytical approach

The Biot-Savart Law, which was used to calculate the induced current, describes the magnetic flux density generated by a coil on any point on the coil's main axis with distance $x$ to the coil's plane. On either side of the axis the distribution of magnetic flux density is Gaussian. The workpiece in the model was not a point but a body with finite size, therefore the flux density varied over the width of the body. To reduce the error following assumptions had to be made:

- The width $w$ of the workpiece had to be much smaller than the radius $r_{Q}$ of the inductor.

- The radius $r_{Q}$ of the inductor had to be smaller than the distance $x$ between inductor and workpiece.

- The thickness of the workpiece had to be smaller than the penetration depth

Also it was assumed that all parameters, except for the resistivity of the workpiece, were not dependent on temperature and magnetic field strength. This 
assumption is justified by the comparison with experiments reported below. However the percentage change in resistivity is probably the largest of the parameters $\left(\sim 117 \%\right.$ over $\left.300{ }^{\circ} \mathrm{C}\right)$. For example the change of the workpiece's width is likely to be a few percent over the temperature range of $300^{\circ} \mathrm{C}$.

For the numeric modelling COMSOL multiphysics software was used. The COMSOL model replicated Fig. 3 and the assumptions of the analytical approach. The thickness of the workpiece was chosen to be half its width.

\subsection{Experimental Validation}

For the experiments an AXIO 10/450 induction heater from Hüttinger Elektronik was used. The generator had a maximum output power of $10 \mathrm{~kW}$ and with the use of a flat helix coil $(\mathrm{d}=80 \mathrm{~mm}, 5$ windings) the operating frequency was about 220 $\mathrm{kHz}$. A steel foil supported in air with a size of 100 $\mathrm{mm} \times 100 \mathrm{~mm}$ and $100 \mu \mathrm{m}$ thickness was heated while non-contact temperature measurements were made with a Flir ThermaCam which was placed above the setup focussing on the foil. The material in the camera software was set to be steel so that the right emissivity was chosen automatically. Steel was chosen as susceptor as it was very easy to handle due to being a rigid and not harmful material with a good performance in electromagnetic fields.

\section{Results \& Discussion}

\subsection{Derivation of Analytical Model}

For a workable process control of the heat dissipation in the workpiece is a key issue. Processing parameters that can be varied include frequency, the heating time, output current, coil to work piece separation and the coil design. Earlier work has shown the basic relationship between those parameters and heat dissipation but a more detailed model is needed to be able to control the process.

Electromagnetic heating is a very complex process and the amount of heat generated in the workpiece depends on many different known and unknown parameters. The resistivity of the susceptor changes with temperature and its relative permeability with the strength of the magnetic field. The strength of the magnetic field outside the coil is not homogenous and also is affected by many parameters like susceptor design, position of the workpiece in the field, and other susceptors (e.g. the workbench) in its range. Shape, design and purity of the susceptor affect the flow of eddy currents and different cooling effects such as heat transfer, heat conduction and radiation apply as well. These factors are not taken into account and hence the model can only be an approximation to predict the applicability and the behaviour of a susceptor.
The power dissipated in a conductor is described with Joule's law:

$P=R \cdot I_{\text {ind }}^{2}$

Where

$P$ : $\quad$ Power dissipated in the conductor

$R: \quad$ Resistance of the conductor

$I_{\text {ind }}$ : Current induced in the conductor

With the equation for quantity of heat being

$Q=P \cdot t$

Where

$Q: \quad$ Heat quantity

$P$ : Power

$t$ : Time

the change of temperature can be described using the induced current and heating time as shown in equation 9:

$d T=\frac{Q}{C p \cdot m}=\frac{R \cdot I_{\text {ind }}^{2} \cdot t}{C p \cdot m}=\frac{\rho \cdot \frac{l}{A} \cdot I_{\text {ind }}^{2} \cdot t}{C p \cdot m}$

Where

$d T$ : Change of temperature

$[\mathrm{K}]$

$R$ : Resistance of conductor

$C p$ : Heat capacity of conductor

$m$ : Mass of conductor

$[\mathrm{J} /(\mathrm{kgK})]$

$\rho:$ Resistivity

$[\mathrm{kg}]$

$l$ : $\quad$ Length of conductor

$A$ : Cross section area of conductor

$[\Omega \mathrm{m}]$

$[\mathrm{m}]$

$\left[\mathrm{m}^{2}\right]$

The only unknown in equation 9 is $I_{\text {ind }}$. As the resistance of the conductor is known $I_{\text {ind }}$ can be replaced if $U_{\text {ind }}$ is known as well.

The voltage induced in a coil can be calculated with

$U_{\text {ind }}=-N \frac{d \phi}{d t}$

Where

$U_{\text {ind }}$ : Induced voltage

$N$ : Number of windings (secondary) coil

$\Phi$ : Magnetic flux through a single winding

$t$ : Time

Using the equation for the magnetic flux
$\phi=\mu \cdot H \cdot A_{a}$
(11) with
$B=\mu \cdot H$
$\mu=\mu_{0} \cdot \mu_{r}$
(13), and 
$\mu_{r}=\frac{B}{B_{0}}$

Where

$\mu$ : $\quad$ Permeability

$H$ : Magnetic field intensity

$A_{a}$ : "Active" area (in magnetic field)

$\mu_{0}$ : Magnetic field constant

$\mu_{r}:$ Relative permeability

$B$ : Magnetic flux density in the conductor $\left[\mathrm{Vs} / \mathrm{m}^{2}\right]$

$B_{0}$ : Magnetic flux density in vacuum $\quad\left[\mathrm{Vs} / \mathrm{m}^{2}\right]$

in equation $10 U_{\text {ind }}$ can be described as followed:

$U_{\text {ind }}=-N \frac{d B}{d t} \cdot A_{a} \cdot \cos \left(\vec{B}, \overrightarrow{A_{a}}\right)$

The magnetic flux density generated by a coil / loop is described in the Biot-Savart law:

$$
B_{o(x)}=n \cdot \frac{I \mu_{0}}{2} \cdot \frac{r_{Q}^{2}}{\left(r_{Q}^{2}+x^{2}\right)^{\frac{3}{2}}}
$$

Where

$B_{0}$ : Magnetic flux density in air

$n$ : Number of windings (primary) coil

I: Output current

$r_{Q}:$ Radius of loop

$x$ : Distance from conductor plane

$[\mathrm{Vs} / \mathrm{m}]$

$[\mathrm{m}]$

From (14), (15), and (16) follows:

$U_{\text {ind }}=-N \cdot n \cdot \frac{d I}{d t} \cdot \frac{\mu_{0} \cdot \mu_{r}}{2} \cdot \frac{r_{Q}^{2}}{\left(r_{Q}^{2}+x^{2}\right)^{\frac{3}{2}}} \cdot A_{a} \cdot \cos \left(\vec{B}, \overrightarrow{A_{a}}\right)$

Using this relationship in equation 9 the change of temperature can be described with known parameters:

$d T=\frac{\left\{N \cdot n \cdot \frac{d I}{d t} \cdot \frac{\mu_{0} \cdot \mu_{r}}{2} \cdot \frac{r_{Q}^{2}}{\left(r_{Q}^{2}+x^{2}\right)^{\frac{3}{2}}} \cdot A_{a} \cdot \cos \left(\vec{B}, \overrightarrow{A_{a}}\right)\right\}^{2} \cdot t}{\rho \cdot \frac{l}{A} \cdot C p \cdot m}$

Assuming the number of windings of coil and workpiece is one $(N=n=1)$ and the vector of the magnetic flux density $B$ being perpendicular to the active area $A_{a}$ a simplified equation can be used:

$d T=\frac{\left\{\frac{d I}{d t} \cdot \frac{\mu_{0} \cdot \mu_{r}}{2} \cdot \frac{r_{Q}^{2}}{\left(r_{Q}^{2}+x^{2}\right)^{\frac{3}{2}}} \cdot A_{a}\right\}^{2} \cdot t}{\rho \cdot \frac{l}{A} \cdot C p \cdot m}$
The generator's output current switches from the positive value of the set current to the negative value and back in a given time which depends on the frequency. We can approximate

$\frac{d I}{d t}=4 \cdot I \cdot f$

it follows that:

$d T=\frac{4 \cdot I^{2} \cdot f^{2} \cdot \mu_{0}^{2} \cdot \mu_{r}^{2} \cdot A_{a}^{2} \cdot t \cdot r_{Q}^{4} \cdot A}{\rho \cdot l \cdot C p \cdot m \cdot\left(r_{Q}^{2}+x^{2}\right)^{3}}$

As mentioned above for ferromagnetic materials a heating effect by hysteresis loss occurs as well. The heating power can be calculated using following equation:

$P_{h y s}=V \cdot f \cdot \int_{0}^{H} B_{(H)} d H=V \cdot f \cdot \frac{\mu \cdot H^{2}}{2}$

Where

$P_{\text {hys }}$ : Heating power by hysteresis loss

[W]

$V$ : Volume of workpiece

$\left[\mathrm{m}^{3}\right]$

$f$ : Frequency

Using equations 12,14 and 16 in equation 22 the change of temperature due to hysteresis alone can be calculated:

$d T_{h y s}=\frac{V \cdot f \cdot \mu_{0} \cdot \mu_{r} \cdot I^{2} \cdot r_{Q}^{4} \cdot t}{8 \cdot\left(r_{Q}^{2}+x^{2}\right)^{3} \cdot C p \cdot m}$

Adding the result of equation 23 to equation 21 the change of temperature can be calculated for ferromagnetic materials as follows:

$d T_{\text {ferro }}=\frac{I^{2} \cdot f \cdot \mu_{0} \cdot \mu_{r} \cdot t \cdot r_{Q}^{4} \cdot A}{C p \cdot m \cdot\left(r_{Q}^{2}+x^{2}\right)^{3}} \cdot\left(\frac{4 \cdot f \cdot \mu_{0} \cdot \mu_{r} \cdot A_{a}^{2}}{\rho \cdot l}+\frac{l}{8}\right)$

Equation 24 leads to the conclusion that for small susceptors, as being used for this project, the amount of heating by eddy currents is big compared to that of hysteresis heating. Even without considering the permeability, which can be above 1000 for steel, for our model hysteresis heating is about a factor $10^{4}$ smaller than eddy current heating.

\subsection{Validation of the Analytical Model}

To validate equations 21 and 24 the functional dependence of $d T$ on $t, x$ and $I$ was compared with that observed in earlier experiments (Knauf et al. 2008). Unfortunately the frequency of the induction current was fixed in the experimental setup used. It therefore was not possible to distinguish between 
eddy current heating (equation 21) and eddy current + hysteresis heating (equation 24).

\subsubsection{Comparison with experiments}

A linear behaviour of heat generation against heating time was observed, while the change of temperature was decreasing non-linear with the $\mathrm{X}$-axis as asymptote against change of working distance and increasing non-linear against variation of the output current. Those relationships are evidently reproduced in equations 21 and 24 .

For more detailed comparison between experiment and the analytical model output current, frequency and radius of inductor coil were chosen to be similar to the experimental parameters. Working distance and susceptor size followed the assumptions for the analytical approach and the material parameters were those of copper, as material properties of the used steel were not available. As the relative change of temperature had to be predicted rather than estimating an absolute change of temperature equations 21 and 24 still could be validated when using material properties different from those of the experiments. The following parameters were used:

Table 1 Parameters - Analytical approach

\begin{tabular}{|c|c|c|c|}
\hline Output current & I & {$[\mathrm{A}]$} & 300 \\
\hline Frequency & $f$ & {$[\mathrm{~Hz}]$} & 200000 \\
\hline \multicolumn{4}{|l|}{ Radius of loop (inductor } \\
\hline coil) & $r_{Q}$ & [m] & 0,02 \\
\hline Distance conductor - loop & $x$ & [m] & 0,03 \\
\hline "Active" area & $A a$ & {$\left[\mathrm{~m}^{2}\right]$} & $5,03 \mathrm{E}-05$ \\
\hline Length of conductor & l & [m] & 0,0125664 \\
\hline $\begin{array}{l}\text { Cross section area of } \\
\text { conductor }\end{array}$ & $A$ & {$\left[\mathrm{~m}^{2}\right]$} & $1,60 \mathrm{E}-05$ \\
\hline Mass of conductor & $m$ & {$[\mathrm{~kg}]$} & 0,0017935 \\
\hline $\begin{array}{l}\text { Heat capacity of } \\
\text { conductor }\end{array}$ & $C p$ & {$\left[\mathrm{~J} /\left(\mathrm{kg}^{*} \mathrm{~K}\right)\right]$} & 340 \\
\hline Resistivity of conductor & $\rho$ & {$[\Omega \mathrm{m}]$} & $3,03 E-08$ \\
\hline Density of conductor & $\rho$ & {$\left[\mathrm{kg} / \mathrm{m}^{3}\right]$} & 8920 \\
\hline Magnetic field constant & $\mu_{0}$ & [Vs/Am] & $1,26 \mathrm{E}-06$ \\
\hline Temperature coefficient & $\alpha$ & {$[1 / \mathrm{K}]$} & 0,0039 \\
\hline Relative permeability & $\mu_{r}$ & [1] & 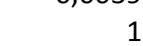 \\
\hline
\end{tabular}

Assuming no other parameter is affected when heating time, working distance or output current, respectively, are varied simplified relations between change of temperature and the varied parameter can be derived from equation 21 .

If heating time is varied while all other parameters are constant the following relation can be assumed:

$d T=X \cdot t$

$\log (d T)=\log (X)+\log (t)$

where $X$ replaces all constant parameters. Plotting $\log (d T)$ of the temperature measured in preceding experiments against $\log (t)$ the trendline should have a slope of 1. As shown in Fig. 4 this was the case:

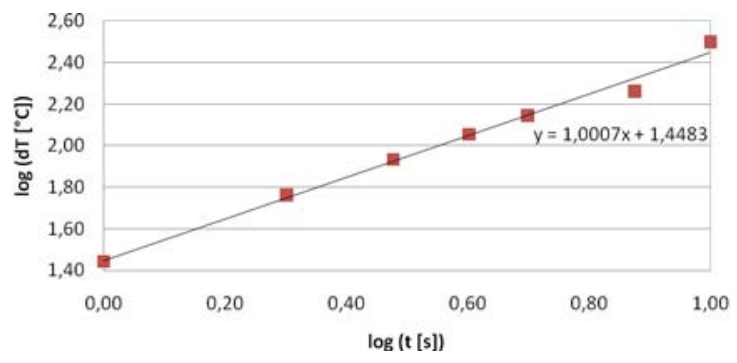

Fig. 4 Measured change of temperature against heating time. The line is a least squares fit, the equation of which is on the graph.

The relation between change of temperature and working distance is rather complex. According to equation $21 d T$ should be constant for $x<<r_{Q}$ and proportional to $\mathrm{x}^{-6}$ for $x>>r_{Q}$. Hence, the slope of the trendline should have a value between 0 and -6 when $\log (d T)$ was plotted against $\log (d)$.

For the experiments a flat helix coil with maximum radius of $40 \mathrm{~mm}$ was used and the working distance was varied from 25 to $50 \mathrm{~mm}$. An intermediate slope (between 0 and -6 ) was expected because working distance and radius were in the same order. As shown in Fig. 5 the trendline had a slope of about -4 :

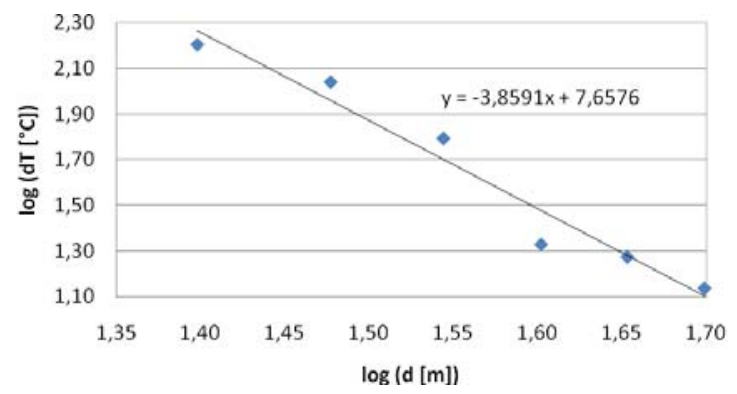

Fig. 5 Measured change of temperature against working distance. The line is a least squares fit, the equation of which is on the graph.

In a final step the relation between output current and change of temperature was derived.

$d T=X \cdot I^{2}$

$\log (d T)=\log (X)+2 \cdot \log (I)$

According to equation 28 the plot $\log (d T)$ vs. $\log (I)$ should have a slope of 2. Fig. 6 shows the plot of two experiments at different working distances: 


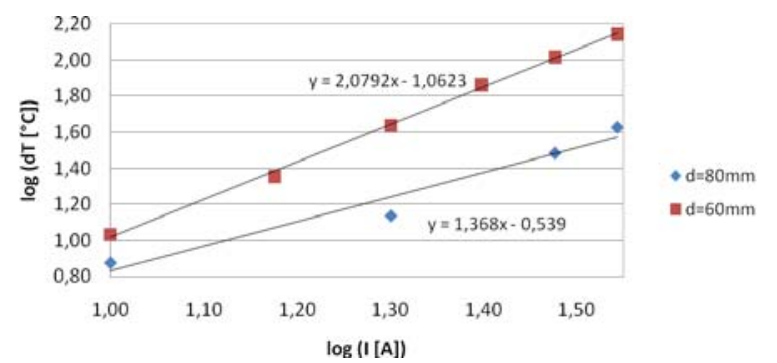

Fig. 6 Measured change of temperature against output current. The lines are least squares fits, the equations of which are on the graph.

The graph for the temperatures generated at a working distance of $60 \mathrm{~mm}$ shows the expected slope of 2 while the temperatures generated at a working distance of $80 \mathrm{~mm}$ did not show the expected behaviour. This is due to inaccuracy of the measurement and a lack of measurement points during the experiments at $80 \mathrm{~mm}$ distance.

Usually the standard deviation $\sigma$ gives an idea of how accurate a calculated slope $b$ of a trendline is. However, if the number of measurements is small a 95\% confidence interval should be applied. Using the $t$ distribution, the uncertainty in the slope can be quantified:

$$
\beta=b \pm t_{95 \%, v} \cdot \sigma_{b}
$$

\section{Where}

$\beta$ : $\quad$ confidence interval of slope $b$

$b$ : $\quad$ slope of trendline

$t_{95 \%, v}$ : critical $t$ value at $95 \%$ confidence level

$\sigma_{b}: \quad$ standard deviation in $\mathrm{b}$

For the experiments at $80 \mathrm{~mm}$ working distance there were only 4 data points which led to a high critical $t$ value of 4.303 . With a standard deviation $\sigma_{b}$ of 0.22 an error of \pm 0.93 was calculated. Hence, the slope could vary between 0.61 and 2.47.

At a working distance of $60 \mathrm{~mm}$ more data points where recorded. Thus, the critical $t$ value was smaller with 2.776 . The error for the slope was calculated to be 0.14 giving a $95 \%$ confidence interval of 1.94 to 2.22 .

\subsubsection{Comparison with simulations}

As mentioned above it is almost impossible to calculate the exact amount of heat that is developed in a workpiece. Engineers from the induction heating equipment manufacturer Hüttinger Elektronik confirmed to the authors that even simulation programs especially designed for induction heating only yield plausible results for very simple designs of coil and workpiece. Nevertheless equation 21 was compared to results from a numerical (COMSOL) model because the numerical model will be used to estimate the heating effect of different susceptor materials and designs and to take into account field and temperature varying parameters in future.

A model was designed using COMSOL following the simplifications of the analytical approach. The parameters of table 1 were used in the model to allow comparing the calculations with simulated results directly:

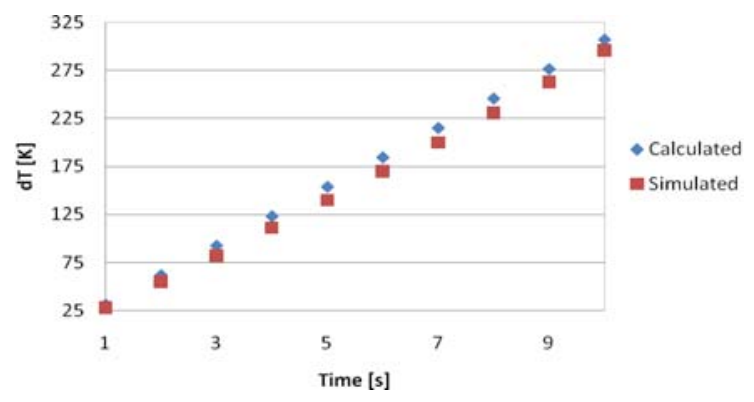

Fig. 7 Comparison of calculated and simulated

temperature change

The calculated and numerically modelled temperatures are almost the same over the range of heating times used. This is also the case for variation of the output current and the working distance, respectively, as long as the working distance is larger than the radius of the inductor (see assumptions for analytical model). When more complex situations with a more realistic coil and workpiece are simulated the numerical model temperatures differ markedly from the analytical model. However, the functional relationships remain the same.

\subsection{Change of Resistivity}

As resistivity changes with temperature a damping of induced voltage could be observed for some simulations which take account of this effect compared to the predictions in equation 21 , which does not.

To calculate the resistivity / resistance in dependency on temperature the temperature coefficient $\alpha$ is used:

$\rho_{(T)}=\rho_{r e f}\left(1+\alpha\left(T-T_{r e f}\right)\right)=\rho_{r e f}(1+\alpha \cdot d T)$

Where

$\alpha$ : Temperature coefficient

Using equation 30 in equation 21 and integrating over time the following relationship can be derived:

$$
d T=\frac{4 \cdot I^{2} \cdot f^{2} \cdot \mu_{0}^{2} \cdot \mu_{r}^{2} \cdot A_{a}^{2} \cdot t \cdot r_{Q}^{4} \cdot A}{\rho_{r e f}\left[1+\left(\frac{\alpha}{2}\right)^{\frac{1}{4}} \cdot d T\right] \cdot l \cdot C p \cdot m \cdot\left(r_{Q}^{2}+x^{2}\right)^{3}}
$$


To compare the heating rate with and without change of resistivity the parameters from Table 1 were used. For temperature dependant resistivity every time step was calculated separately. As the change of temperature $d T$ is found on both sides of the equation it was varied in an iterative fit until equation 31 was true.

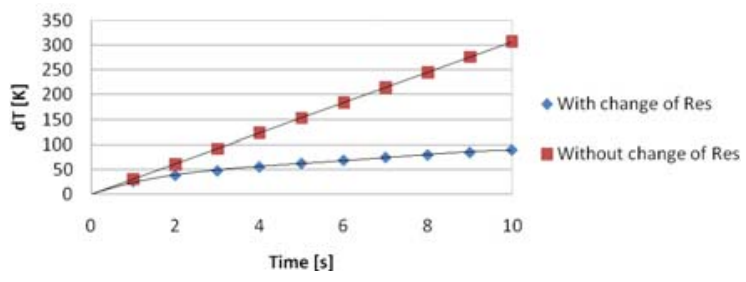

Fig. 8 Calculated temperature change with and

without change of resistivity

As shown in Fig. 8, a significant damping effect may be observed. The change of temperature shown in Fig. 4 seems to be linear. This is due to the measurements starting after one second of heating and the relative permeability $\mu_{r}$ of steel being 40 to 7000 times higher than the one of copper, which was used for the analytical model. To summarise, equations 21 and 31 are of use in describing the dependence of temperature on the process parameters rather than predicting real temperatures. While equation 31 takes into account more physics, the simpler equation 21 seems to describe experimental observations well.

\subsection{High vs. Low Resistivity}

Equation 2 may lead to the conclusion that higher resistivity of the susceptor material leads to higher heat generation for a given coil current. But as the current flowing in a workpiece is a result of the induced voltage its value depends on the resistivity. Hence the amount of heat generated in the workpiece is inversely proportional to the resistivity as shown in equations 21 and 31 . The dependence of current on resistivity was studied using the COMSOL model.

All parameters were kept constant while the resistivity of the workpiece was altered. The dimensions of the workpiece were in the range of the penetration depth to guarantee a homogeneous current distribution. Both, current density and generated heat were simulated:

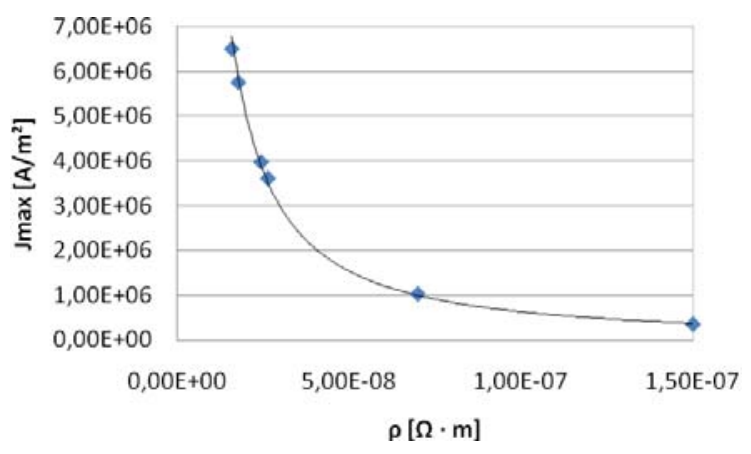

Fig. 9 Maximum workpiece current density against resistivity from COMSOL simulation. The line is a guide to the eye.

The current density variation with resistivity is shown in Fig. 9. As expected the temperature change showed similar behaviour against resistivity as shown in Fig. 10:

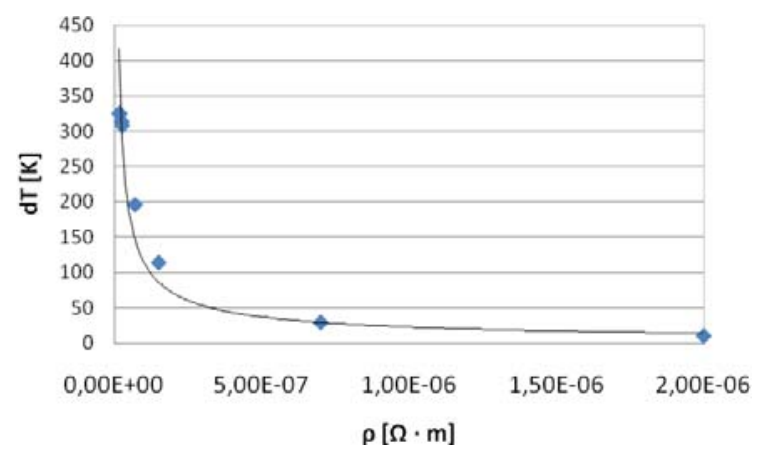

Fig. 10 Change of temperature against resistivity

from COMSOL simulation. The line is a guide to the eye.

Thus the higher the conductivity of a material the better it works as susceptor for induction heating.

\subsection{Cooling Effects}

During induction heating the workpiece is not only heated but cooling effects occur as well. At equilibrium the cooling power equals the heating power. Ignoring other effects like phase changes (e.g. when the susceptor starts to melt) the temperature at equilibrium is the expected maximum achievable for a particular setup. Three main cooling effects have to be considered.

If a solid body is surrounded by or has contact to a liquid or gaseous medium of a different temperature heat is transferred from the warmer to the cooler element. This effect is referred to as heat convection. Equation 32 describes the power of the transferred heat:

$$
P_{h t}=\alpha_{h t} \cdot A_{h t} \cdot\left(T_{S}-T_{M}\right)
$$


Where

$P_{h t}$ : Power of transferred heat

$\alpha_{h t}$ : Heat transfer coefficient

$A_{h t}$ : Contact area

$T_{S}$ : Temperature solid

$T_{M}$ : Temperature medium

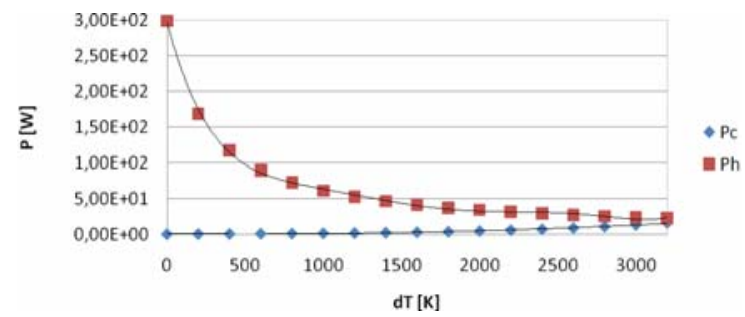

Fig. 11 Calculated balance of heating and cooling

Another effect is heat conduction. If there are different temperatures within a heat conductor there is a heat flux from the warmer to the cooler area to restore heat equilibrium.

In the joining application the plastic substrate works as heat conductor transporting heat away from the interface between substrate and susceptor. The power of conducted heat can be calculated using equation 33:

$P_{h c}=\lambda \cdot \frac{A_{h c}}{s_{h c}} \cdot\left(T_{A}-T_{B}\right) \quad$, with $T_{A}>T_{B}$

Where

$\Delta Q_{h c}$ : Quantity of conducted heat

$\lambda$ : $\quad$ Coeff. of thermal conductivity

$A_{h c}: \quad$ Cross sectional area

$S_{h c}$ : Length of heat conductor

$[\mathrm{W} /(\mathrm{Km})]$

$\left[\mathrm{m}^{2}\right]$

$T_{A}, T_{B}$ : Temperatures

$\Delta t$ : Time interval

The third effect to be considered is heat radiation. While radiating heat a body cools down. The power of radiated energy can be calculated with the StefanBoltzmann law for grey bodies:

$P_{h r}=\varepsilon_{(T)} \cdot \sigma \cdot A_{h r} \cdot T_{a}^{4}$

Where

$\sigma$ : Stefan-Boltzmann constant

$A_{h r}$ : Surface area of radiator

$T_{a}$ : Absolute temperature of radiator

$\left[\mathrm{W} /\left(\mathrm{m}^{2} \mathrm{~K}^{4}\right)\right]$

$\varepsilon_{(T)}$ : Emissivity

In the experiments and models the susceptor is supported in air and not attached to a substrate. Hence, the cooling by heat conduction can be assumed to be negligible.

In Fig. 11 the sum of equations 32 and 34 are plotted together with the heating power, for the parameters in Table 1 and assuming an emissivity of 0.015 (for copper). It can be seen that power balance is reached at a temperature change above $3000 \mathrm{~K}$.

\section{power in air}

As mentioned before this calculation ignores changes in material properties depending on temperature (except change of resistivity). Nevertheless we can conclude that air cooling effects do not seem to be very important for temperatures below $500 \mathrm{~K}$, where the polymer bonding processes take place. Of course during bonding the susceptor is surrounded by plastic so air cooling does not apply at all. If we calculate heat flow in an ideal situation where there is no thermal barrier between susceptor and surrounding material and the outer surface of the plastic is kept at a constant temperature we find the power magnitude of cooling by conduction can be less (for thick plastic sheets) or more (for thin plastic sheets) than by convection. But even if the cooling power by conduction was some ten times higher than cooling by convection, it still would be much smaller than the heating power for the desired operating temperatures of the process of $d T<200 \mathrm{~K}$. It therefore seems likely that to model plastics joining the temperature of the susceptor can be calculated without considering heat dissipation into the plastic, at least while the induction field is present. This would allow magnetic effects (susceptor heating) to be considered separately from thermal effects on the plastic (size of heat affected and melted zones) in numerical modelling, simplifying the models. The modelling of thermal effects on the plastic is the subject of further investigation.

\section{Conclusions \& Future Work}

Analytical equations describing the heating of a susceptor in an induction field were derived for a simplified situation, to provide the basis for process optimisation and design rules for the low frequency induction heating (LFIH) plastics joining technique. The equations predict a linear behaviour of heat generation against heating time, decreasing nonlinear dependence against increase of working distance and superlinear increase with increasing generator coil current. The predictions were consistent with the results of experiments and simulations previously published by the authors.

Cooling effects were considered in this work in association with the heating. Calculations, although not taking account of some changes of material properties, have shown that those effects are 
negligible when working at low temperatures $(<500$ $\mathrm{K})$.

To summarise the findings, the guidelines can be drawn up for the design of susceptors:

- Materials with low resistivity perform better

- Materials with high permeability perform better

- The cross section area of the susceptor should be as large as possible to reduce resistance

- The thickness of the susceptor should be in the dimensions of the penetration depth or smaller to increase homogeneity of heat dissipation

- The shape of the susceptor should follow the shape of the inductor coil or vice-versa to increase homogeneity of heat dissipation.

Further work will cover susceptor shape effects, and thermal effects and the size of the heat affected zone in plastics to be joined.

\section{Acknowledgments}

This work was sponsored by the EPSRC Grand Challenge project 3D-Mintegration.

\section{References}

Anonymous (1993) Induction Heating Technology. EPRI Center for Materials Fabrication, Columbus, Ohio. TechCommentary. vol. 2.

J. Callebaut (2007) Induction Heating. Power Quality \& Utilisation Guide.

Cheltenham Induction Heating Ltd (2006) Tamper-

Evident Cap Sealing.

S. C. Chen, W. R. Jong, Y. J. Chang, J. A. Chang and J. C. Cin (2006) Rapid mold temperature variation for assisting the micro injection of high aspect ratio micro-feature parts using induction heating technology. J Micromech Microengineering. vol. 16. pp. 1783-1791.

J. Davis and P. Simpson (1979) Induction Heating Handbook. London; New York: McGraw-Hill. p. 310.

B. J. Knauf, D. P. Webb, C. Liu and P. P. Conway (2008) Packaging of polymer based microfluidic systems using low frequency induction heating (LFIH). Electronic Packaging Technology \& High Density Packaging. pp. 1-6.

B. J. Knauf, D. P. Webb, C. Liu and P. P. Conway (2008) Plastic Packaging Using Low Frequency Induction Heating (LFIH) For Microsystems. Electronics Packaging Technology Conference. pp. 172-180.

R. J. Nichols, D. P. LaMarca and B. Agosto (2006) Performance of Susceptor Materials in High Frequency Magnetic Fields. Proc. Of ANTEC, SPE. V. K. Stokes (2003) Experiments on the induction welding of thermoplastics. Polymer Engineering \& Science. vol. 43. pp. 1523-1541.

H. A. Yang, M. Wu and W. Fang (2005) Localized induction heating solder bonding for wafer level
MEMS packaging. J Micromech Microengineering. vol. 15. pp. 394-399.

H. A. Yang, C. W. Lin, C. Y. Peng and W. Fang (2006) On the selective magnetic induction heating of micron scale structures. J Micromech Microengineering. vol. 16. pp. 1314-1320. S. Zinn and S. L. Semiatin (1988) Coil design and fabrication: basic design and modifications. Heat Treating. pp. 32-41. 